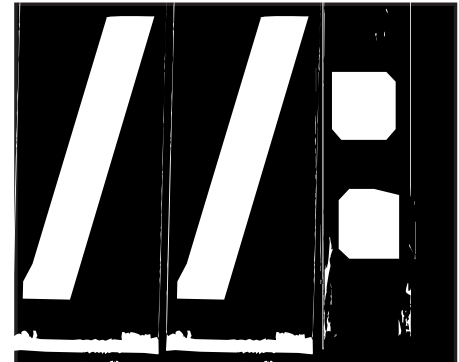

aduca"ción y comunicación 11: 111-118 Nov. 2015

\section{EL APRENDIZAJE ELECTRÓNICO MÓVIL: ENTRE LA OPORTUNIDAD Y EL RIESGO. UNA REFLEXIÓN CRÍTICA}

\section{Mobile learning between opportunities and risks. A critical thinking}

\author{
Davide Borrelli \\ Universidad Suor Orsola Benincasa de Napoles (Italia) \\ E.mail: davide.borrelli@unisalento.it \\ Carmine Piscopo \\ Università Sapienza de Roma (Italia) \\ E.mail: carmine.piscopo@gmail.com
}

Resumen:

Muchas veces se concibe la introducción de tecnologías digitales en las instituciones educativas de forma acrítica, como una panacea en grado de mejorar por sí misma las prácticas y potenciar el rendimiento. Tales expectativas se fundan en una visión que considera las tecnologías como un factor autónomo y aislado, independientemente de las actividades para las cuales se emplean, de su significado y de las expectativas que el usuario les atribuye, además de las relaciones sociales y los contextos culturales y organizativos en las cuales son introducidas y utilizadas. El riesgo que se presenta para las tecnologías de aprendizaje electrónico móvil (m-Learning) es que sean objeto de la misma parcialidad interpretativa. La finalidad de nuestro artículo es presentar las aplicaciones de las mencionadas tecnologías, así como sus perspectivas de desarrollo.

Palabras clave: M-learning, educación superior, universitaria digitalizada, medios digitales, escenario de medios móviles

\title{
Abstract:
}

Often the introduction of the digital technologies in higher education is considered in itself as a panacea capable of improving its performance. These expectations are based on a theoretical framework that considers the technologies as isolated and autonomous factors, regardless of the activities for which they are employed and independently of the meaning and expectations that its users will perceive as well as the socio-cultural and organizational contexts where they are introduced and used. We are facing the risk that also mobile learning technology could be object of this interpretative bias. Our article aims to present its applications and development prospects.

Keywords: m-learning, higher education, digitized university, digital media, mobile media scenario

Recibido: 27-07-2015 Revisado: 28-08-2015 Aceptado: 25-09-2015 Publicado: 01-11-2015

https://dx.doi.org/10.25267/Hachetetepe.2015.v2.i11.10 


\section{II: Educación y comunicación actual}

\section{Introducción}

$\mathrm{L}$ as tecnologías digitales móviles han rediseñado en tan poco tiempo tantos aspectos de la vida social (cfr. Orton-Johnson and Prior, 2013) y disparado procesos de mutación tan profundos y complejos, que aún resulta difícil identificar sus límites y estimar claramente el alcance de dichas transformaciones. Dichas tecnologías son para nosotros como el agua para un pez, o como afirma McLuhan: son el "ambiente" (Peters, 2015) en el cual vivimos y del cual no podremos nunca separarnos, aun cuando no somos capaces de ser completamente conscientes de su existencia, justamente por el hecho de estar inmersos en ese ambiente. Consecuentemente, era de esperar que los procesos formativos fuesen también alcanzados por la ola de innovaciones tecnológicas.

El presente artículo tiene por objeto analizar particularmente los factores ambivalentes, y a veces problemáticos, del aprendizaje electrónico móvil. El ensayo recorre sintéticamente el sentido y la experiencia de las tecnologías de la comunicación móvil, evidenciando posibles conexiones con el campo educativo, sugiriendo finalmente una reflexión sobre el rol que estas tecnologías se proponen jugar en los procesos de digitalización de la educación superior, e incluso en las oportunidades y problemas que dicha transformación comporta en la redefinición de la misión histórica de la Universidad.

\section{Una revolución tecnológica para todos}

La tecnología móvil hizo su aparición en nuestra sociedad en los años 90, insertándose en un nicho de mercado correspondiente a una porción muy específica del mundo del trabajo, ofreciendo un servicio de prestigio a costes sólo accesibles para unos pocos usuarios. Como sucede frecuentemente con la tecnología, en pocos años y gracias a un intenso proceso de democratización empujado fuertemente por las grandes empresas de telecomunicaciones, el teléfono móvil ha llegado a expandirse a todas las categorías de usuarios: desde los más jóvenes hasta los ancianos, desde el obrero al director de empresa. A mitad del año 2000, muchos países (cfr. http:// data.worldbank.org) ya habían alcanzado e incluso superado la barrera del $100 \%$ de penetración: esos países tenían ya más teléfonos móviles que habitantes. La consecuencia más obvia es de origen económico: el mercado de la telefonía móvil ha crecido al punto de haberse transformado en un nuevo negocio, abriendo nuevas oportunidades de desarrollo comercial. Nacen los llamados VAS (Value Added Services), que buscan explotar plenamente las posibilidades que el hardware y la conexión telefónica ofrecen.

El principal problema que obstaculizaba un desarrollo real de ésta tecnología estaba representado justamente por la limitada fruición del contenido: pantallas demasiado pequeñas, resolución de vídeo insuficiente y de baja calidad de color, pero sobre todo, una conexión de Internet demasiado lenta. Es con la llegada de la tecnología de red $2 \mathrm{G}$ y la introducción del primer modelo de iPhone (2007), cuando el escenario del mercado móvil como lo conocemos hoy toma forma. Desde ese momento, el avance de la tecnología en general y de la tecnología móvil en particular se propaga en manera imparable, agregando millones de nuevos usuarios por año.

En un período menor a diez años, se ha hecho posible hablar de una dependencia casi completa del teléfono 


\section{I: El aprendizaje electrónico movil: Entre la oportunidad 11: y el riesgo. Una reflexión crítica}

móvil, consecuentemente con una oportunidad en términos de crecimiento económico y desarrollo de nuevas ideas. La tecnología móvil ha conquistado una gran parte de la población mundial. Para comprender las proporciones del fenómeno, utilizaremos los datos publicados por "We are social" (2015) en enero de 2015. Los usuarios de Internet son cerca de 3 billones, con una tasa de penetración a nivel mundial del $42 \%$, mientras las cuentas en redes sociales llegan al 29\% de la población mundial. Los usuarios de telefonía móvil son cerca del 51\%, mientras son $23 \%$ los usuarios que utilizan el móvil para navegar los sitios de social media. A nivel europeo las estadísticas muestran incrementos notables: basta considerar que la penetración del teléfono móvil es del 132\%, mientras que los usuarios de Internet abarcan el 70\% de la población y las cuentas de redes sociales llegan al $46 \%$, siendo de $34 \%$ el uso de las últimas desde móviles.

Figura 1 - Global digital snapshot

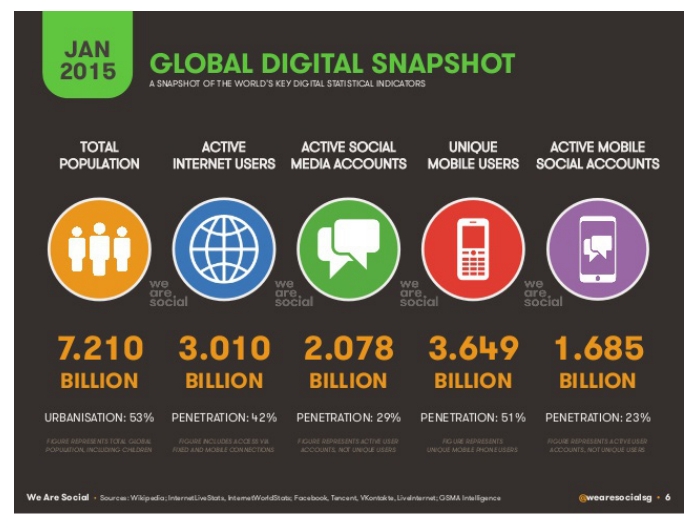

We are social, (2015), Digital, Social \& Mobile in 2015.

\section{La obligación de lo nuevo: las competencias tecnológicas de los nuevos usuarios}

Frente a una presunta democratización de la conectividad tecnológica, aumentan las necesidades y las competencias de los individuos, por lo cual la vieja cuestión de la brecha digital no tendría que ver tanto con la posibilidad de acceso, como con la calidad del consumo tecnológico. Este fenómeno, incluso antes que describirlo como económico, está íntimamente ligado con la manera en la que el sujeto se relaciona con la tecnología. El tiempo y espacio que ocupa tanto Internet en general, como móviles y tablets en particular, es siempre más amplio y no se restringe ya al ámbito laboral, sino que se extiende también al privado y afectivo. Es fundamental partir del presupuesto de que no estamos frente a una herramienta que se encuentra confinada entre los muros del espacio laboral, por el contrario, hablamos de una tecnología que está cada vez más entre nosotros, e incluso nos sigue a donde vayamos. Obviamente el valor agregado no se puede atribuir solamente a la evolución del hardware (smartphone, tablet, etc.), sino a la posibilidad de aprovechar la conexión de Internet, es decir, a la posibilidad de la conectividad -en sentido amplio- que ella ofrece.

Basándonos a los datos presentados sintéticamente en la primera parte, resulta claro el peso que el uso del móvil tiene en la vida de los individuos. Los nuevos dispositivos móviles permiten cultivar las propias relaciones sociales y las necesidades informativas sin imitaciones de tiempo o espacio, por ejemplo a través de las redes sociales y las aplicaciones relacionadas con las mismas. Lo mismo vale para adultos que para "mayores" muchas veces considerados al margen del 
consumo tecnológico (quienes salieron hace poco tiempo del mundo laboral, después de haber utilizado por años Internet y las nuevas interfaces digitales). Con el hábito de utilizar estas herramientas crece también la habilidad de los usuarios de realizar operaciones complicadas (al menos en apariencia), como por ejemplo en el caso del e-commerce y el m-commerce, fenómenos hoy ampliamente difundidos.

Cabe mencionar que, aumentando la comunicación entre personas, máquinas y servicios, crecen correlativamente los riesgos asociados a ellas. Sobre este punto hay numerosos estudios en la literatura pedagógica internacional, los cuales ofrecen frecuentemente posiciones encontradas. Desde el punto de vista más optimista, se sostiene que el empleo de las tecnologías permite una mayor interacción entre los sujetos, anulando los límites de espacio y tiempo, favoreciendo la creación de comunidades y la difusión de intereses (Rainie and Wellman, 2012). Por otro lado, según las posiciones más negativas, la fuerte dependencia del smartphone puede derivar en un correlativo aislamiento de determinados sujetos (Turkle, 2011), los cuales encuentran en el mundo digital mayor satisfacción que en el mundo real. La diatriba académica es probablemente imposible de conciliar. Sin embargo, las oportunidades y límites de estas tecnologías se conocen cada vez más en la vida cotidiana de los individuos. Así como sucedió con los medios tradicionales de comunicación, la fase de domesticación (Silverstone and Hirsch, 1992) no sólo ha conducido a una completa inclusión de los medios digitales en la esfera privada, sino que ha permitido también la circulación de información asociada a los riesgos que conlleva el uso exacerbado de Internet. La clave para observar e interpretar dichos fenómenos está justamente en el término "red" (Network), que pasa de una concepción estática a otra típica de la "Web 1.0", donde comienza a jugar un rol fundamental la movilidad y las oportunidades que ella ofrece.

En otras palabras, las tecnologías móviles e Internet - cuya accesibilidad se caracteriza por la ubiquidad espacial (permiten un flujo informativo heterogéneo, en el interior del cual el usuario aprende rápidamente a moverse con fluidez entre los distintos campos). Entre éstos, el sector de la enseñanza resulta ser uno de los más interesantes, fundamentalmente si se piensa en las perspectivas futuras que plantea.

A partir de Livingstone (2012), quien sostiene la importancia del rol de las tecnologías relacionadas con la enseñanza en contextos institucionales tanto públicos como privados, el m-Learning viene frecuentemente descrito como una oportunidad de aprendizaje sin vínculos físicos (Kukulska-Hulme 2005) que, como sostiene Pachler (2009: 5), permite a los usuarios "re-interpret their everyday life contexts as potential resources for learning".

Desde cierto punto de vista, poder aprovechar las oportunidades concretas que tal tecnología ofrece más allá del campo del entretenimiento, es un modo inteligente de utilización del móvil. La experiencia del aprendizaje puede enriquecerse con los soportes multimedia, activando lógicas de gamification, en las cuales el usuario interactúa y personaliza el propio recorrido formativo, agregando información específica, imágenes, vídeo, etc.

El m-Learning es una oportunidad para muchas empresas e instituciones interesadas en invertir en el sector de la formación que permite abrir oportunidades de aprendizaje que no gravitan necesariamente sobre el tiempo que sus miembros dedican a las tareas 


\section{I/: El aprendizaje electrónico movil: Entre la oportunidad y el riesgo. Una reflexión crítica}

cotidianas. Ello significa que la tecnología móvil permite a los usuarios ser libres de administrar los módulos didácticos o informativos aprovechando aquellos tiempos considerados inutilizables, como por ejemplo el tiempo destinado al trayecto entre la casa y el lugar trabajo, las pausas del almuerzo, etc.

Para iniciar este nuevo tipo de proceso de aprendizaje que complementa a los métodos clásicos presenciales, no solo es obligatorio un cambio tecnológico, sino que más bien un cambio cultural y social. Las mayores dificultades que se presentan a la hora de encarar estos nuevos trayectos, no ya derivadas del hardware (como podía verificarse antes de 2007) sino de las competencias de las partes involucradas en el proceso formativo: quien aprende $\mathrm{y}$ quien ofrece la información. Aparece aquí un elemento de sustentabilidad (Wan y Howard, 2013), de una nueva infraestructura comunicativa y educativa, que debe necesariamente plantearse desde la óptica del aprendizaje, y por lo tanto desde la esfera educativa. Los usuarios, sobre todo los más jóvenes, ya han demostrado una cierta flexibilidad y ductilidad hacia el nuevo formato multimedia. El verdadero desafío reside en la arquitectura de la oferta, partiendo de las competencias profesionales, e incluyendo la renovación de las estructuras tecnológicas. Las experiencias consolidadas en estos años han resultado positivas, incluso si se encuentran aún en fase inicial. En el futuro cercano, como demuestran los datos relativos a conexiones móviles, el vínculo entre tecnología móvil e individuo se verá reforzado ulteriormente. No aprovechar plenamente este cambio, dejando de proponer un uso que incluya también la oferta formativa, sería una gran pérdida, no sólo en términos de competitividad, sino también en referencia a las posibilidades de generar nuevas formas de socialización en relación con los medios digitales.

\section{El futuro de la universidad digital}

También la educación terciaria empieza a sufrir una transformación radical debido al uso de plataformas digitales y a la adopción de sistemas de m-learning. No es casualidad que en este momento la cuestión de la "universidad digital" sea objeto frecuente de análisis por parte de diferentes especialistas ya sea como tema de reflexión teórica (Goodfellow y Lea, 2013) u objeto de investigación empírica (Lupton, 2015).

En el proyecto de desarrollo de la universidad digital, las soluciones de m-learning no sólo favorecen los procesos de desintermediación formativa que generalmente caracterizan la educación a distancia sino que, además, están destinadas a potenciar en particular otros dos aspectos adicionales: por una parte, permiten una siempre mayor reducción de los costes infraestructurales propios de la actividad formativa; por otra, acentúan la responsabilidad personal de los estudiantes respecto al control y la gestión del momento y el lugar del propio aprendizaje (Kearney et al., 2012). La literatura especializada ha ido analizando paulatinamente los diferentes aspectos que caracterizan las propuestas formativas que pueden ser organizadas a través del m-learning: obviamente, en primer lugar, el uso en situaciones de movilidad y de no residencialidad (Sharples et al, 2009), pero también la facilidad del acceso y la inmediatez de la fruición (Kynäslahti, 2003), así como el valor añadido de la situacionalidad (Cheon et al, 2012) y de 
la seamlessness, es decir, de la contextualidad y de la ausencia de solución de continuidad entre el ambiente de aprendizaje y el ambiente de vida (Wong, Milrad y Specht 2015) en el que transcurre la acción formativa. De cualquier manera, el escenario que el m-learning está delineando no parece anunciar sólo promesas y oportunidades sino también riesgos y potenciales insidias. Al igual que las otras tecnologías, tampoco los sistemas de m-learning pueden ser considerados como un factor autónomo y aislado, al margen de la actividad para la que son utilizados, del significado y de las expectativas que quien los usa les atribuye, así como de las relaciones sociales y de los variados contextos culturales y organizativos en los que son introducidos y puestos en funcionamiento (Selwyn, 2011). En la estructuración intrinsecamente sociocultural y no solamente tecnológica del m-learning, se pueden descubrir las razones que explican algunos efectos ambivalentes que pueden hallarse tanto en la forma que adoptan las instituciones formativas como en las experiencias que experimentan sus destinatarios.

Las universidades digitalizadas tienen la posibilidad de hacer llegar su oferta educativa a sectores de la población situados más allá de las áreas territoriales de competencia y, como consecuencia, el beneficio económico que pueden obtener desarrollando las tecnologías de m-learning tiende a ser claramente más elevado de lo habitual. En otras palabras, el potencial incremento del número de estudiantes no supone solamente un aumento de la credibilidad social de las instituciones educativas sino que además abre nuevos y más amplios espacios de mercado para la educación terciaria, generando la posibilidad de aumentar el rendimiento económico. Todo ello ejerce una fuerte presión que está llevando a una progresiva redefinición de la misión tradicional de la universidad, haciendo prevaler la misión de proveedor de un servicio comercial en detrimento de aquélla tradicional de agente de distribución de un bien público. Naturalmente, no es en sí el desarrollo de plataformas de m-learning las que desencadenan estos procesos de mercantilización de la educación, sino el que operen en el contexto de determinadas lógicas organizativas y en el ámbito de orientaciones y sensibilidades ligadas socio-culturalmente a dichos procesos. La realidad es que, de hecho, el m-learning se presta particularmente a este tipo de resultados ambivalentes.

Análogas ambivalencias pueden encontrarse en la experiencia de los estudiantes. Por ejemplo, si la deslocalización del contexto formativo comporta un potencial aumento del margen de beneficiados y, como consecuencia, una indudable democratización del acceso a los preciados contenidos de la educación superior. También es verdad que la posibilidad que se le ofrece a cada estudiante a distancia de gestionar con relativa autonomía los propios modos y ritmos de aprendizaje tiende a favorecer la polarización del relativo rendimiento académico, y pone las bases para que, en el futuro, la brecha profesional, económica y social entre unos y otros vaya ensanchándose progresivamente. En consecuencia, los estudiantes más capaces y más interesados en los contenidos educativos encontrarán en el m-learning un instrumento ideal con el que perfeccionar sus competencias y probablemente garantizarse el acceso a una mejor ocupación, mientras que aquéllos menos dotados, abandonados a sí mismos y privados del constante estímulo de la presencia del docente, 


\section{I/: El aprendizaje electrónico movil: Entre la oportunidad y el riesgo. Una reflexión crítica}

tendrán dificultades siempre mayores para alcanzar resultados aceptables en el aprendizaje y tenderán, por ello, a ser penalizados en el mercado laboral. Es el escenario, pronosticado entre otros por el economista estadounidense Tyler Cowen, de una "instrucción hipermeritocrática" en la que el m-learning podría desempeñar probablemente el papel de agente igualitario, pero de hecho lo haría de manera muy singular pues, respecto al pasado, eliminaría todas las cualidades y los valores intermedios, ya que "puede catapultar individuos inteligentes, motivados pero no pertenecientes a la élite, por encima de los miembros de comunidades elitarias; pero no puede empujar a un estudiante distraído a los vértices del grupo" (2013: 183 trad. it.).

En conclusión, consideramos que el m-learning constituye un sector de vanguardia particularmente interesante por sus posibilidades para potenciar la oferta educativa y de apertura a un mayor número de destinatarios. Es necesario desarrollar un serio programa de investigación para entender plenamente su eficacia formativa y, sobre todo, para indagar de qué manera está influyendo en la transformación de la naturaleza y el papel de las instituciones educativas. Muchas de estas dinámicas están aún por estudiar y entender a fondo. En particular, señalamos la necesidad de que los estudios sobre las tecnologías de m-learning se concentren sobre todos los aspectos de la universidad en cuanto institución, sobre el análisis de las formas de organización y de gestión que tienden a desarrollarse en el ambiente de la universidad digital, sobre los significados profesionales y personales ligados al uso de tales tecnologías, sobre el tipo de prácticas, prioridades, interpretaciones y orientaciones a ellas asociadas, y que afectan tanto a las instituciones universitarias como al personal docente y a los estudiantes que con ellas trabajan. Se trata de una condición indispensable para poder elaborar una política de la educación superior que, como ha observado Neil Selwyn, apunte a "to reconcile the idea of 'university' for the digital age in a way that retains the core essence of higher education as a public good?" (2014: 7).

\section{Referencias}

Cheon, J., Lee, S., Crooks, S. M. and Song, J. (2012). An Investigation of Mobile Learning Readiness in Higher Education Based on the Theory of Planned Behaviour. Computers \& Education, 59 (3); 10541064.

Cowen, T. (2013). Average is Over. Powering America Beyond the Age of the Great Stagnation. New York: Dutton (trad. it. La media non conta più. Ipermeritocrazia e futuro del lavoro). Milán: Università Bocconi Editore.

Goodfellow, R. and Lea, M. R. (Eds.) (2013). Literacy in the Digital University. Critical Perspectives on Learning, Scholarship and Technology. Londres y Nueva York: Routledge.

Kearney, M., Schuck, S., Burden, K. and Aubusson, P. (2012). Viewing Mobile Learning from a Pedagogical Perspective. Research in Learning Technology, 20 (1); 1-17.

Kukulska-Hulme, A. (2005). Mobile Usability and User Experience, en Kukulska-Hulme, A. and Traxler, J. (Eds.). Mobile learning: A Handbook for Educators and Trainers. Londres: Routledge; 45-56.

Kynäslahti, H. (2003). In Search of Elements of Mobility in Context of Education, en Kynäslahti, $\mathrm{H}$. 


\section{II: Educación y comunicación actual}

and Seppälä (Eds.). Mobile Learning, Helsinki. IT Press; 41-48.

Livingstone, S. (2012). Critical Reflections on the Benefits of ICT in Education. Oxford Review of Education, 38(1); 9-24.

Orton-Johnson K. and Prior, N. (Eds.) (2013). Digital Sociology. Critical Perspectives, Houndmills, Basingstoke. Hampshire y Nueva York: Palgrave Macmillan.

Rainie, L. and Wellman, B. (2012). Networked. The New Social Operating System. Cambridge, MA: The MIT Press.

Lupton, D. (2015). Digital Sociology. Londres y Nueva York: Routledge.

Pachler, N. (2009). Research Methods in Mobile and Informal Learning: Some Issues, en Vavoula, G., Pachler, N. and Kukulska-Hulme, A. (Eds.). Researching Mobile Learning: Frameworks, Tools and Research Designs. Oxford: Peter Lang Publishing; $1-15$.

Peters, J. D. (2015). The Marvelous Clouds. Toward a Philosophy of Elemental Media. Chicago: University of Chicago Press.

Selwyn, N. (2011). Education and Technology. Key Issues and Debates. Londres y Nueva York: Continuum.
Selwyn, N. (2014). Digital Technology and the Contemporary University. Degrees of digitalization. Londres y Nueva York: Routledge.

Sharples, M., Arnedillo-Sánchez, I., Milrad, M., \& Vavoula, G. (2009). Mobile learning: Small devices, big issues, en Ludvigsen, S., Balacheff, N., Jong, T. D., Lazonder, A., and Barnes, S. (Eds.). Technologyenhanced Learning: Principles and Products. Berlin: Springer-Verlag; 233-249.

Silverstone, R. and Hirsch, E. (Eds) (1992). Consuming Tecnologies. Media and Information in Domestic Spaces. Londres: Routledge.

Turkle, S. (2011). Alone Together: Why We Expect More from Technology and Less from Each Other. Nueva York: Basic Book.

Wan, N. and Howard, N. (2013). A Framework for Sustainable Mobile Learning in Schools. British Journal of Educational Technology, Vol 44 No 5 2013; 695-715 (10-09-2015)

We are social, (2015). Digital, Social \& Mobile in 2015. http://wearesocial.net/.

Wong, L-H, Milrad, M., Specht, M. (Eds.) (2015). Seamless Learning in the Age of Mobile Connectivity. Singapur, Heidelberg, Nueva York, Dordrecht y Londres: Springer.

Word Bank, (2015). Mobile cellular subscriptions (per 100 people). http://data.worldbank.org/ (10-09-2015) 